\title{
Terapia con fibrinólisis local dirigida por catéter en tromboembolismo pulmonar de riesgo intermedio
}

\section{Therapy with local fibrinolysis directed by catheter in intermediate risk pulmonary thromboembolism}

\author{
Mauricio Orozco-Levi, Melissa Mogollón-Vargas, \\ Libardo Medina-López, Tamara Gorgadze, Diana Cáceres-Rivera, \\ Rubén Duarte-Reyes, Alba Ramírez-Sarmiento, Carlos Reyes-Martínez, \\ Camilo Pizarro-Gómez, José Federico SaAibi-Solano \\ - Bucaramanga (Colombia)
}

\section{Resumen}

Introducción: en pacientes con tromboembolia pulmonar (TEP) aguda, la fibrinólisis arterial local puede tener una relación muy favorable entre beneficios y riesgos.

Objetivo: caracterizar las condiciones epidemiológicas y clínicas de los pacientes con TEP agudo de riesgo intermedio que han recibido terapia fibrinolítica urgente dirigida por catéter.

Métodos: estudio descriptivo de una serie de casos de pacientes atendidos en la Fundación Cardiovascular de Colombia (período 2012-2016) con diagnóstico de TEP de riesgo intermedio llevados a terapia fibrinolítica mediante catéter pulmonar.

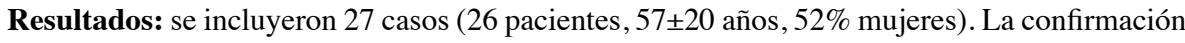
de embolia pulmonar aguda se realizó mediante angiotomografía. El 85\% de los casos mostró signos ecocardiográficos de disfunción ventricular derecha, 74\% elevación de troponinas, y $82 \%$ elevación de BNP o pro-BNP. El tiempo puerta-aguja desde el diagnóstico hasta la fibrinólisis fue de $29 \pm 31$

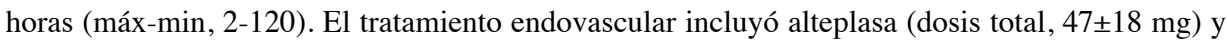
fragmentación-aspiración mecánica mediante catéter pulmonar. El 78\% de los pacientes mostró disminuciones en la PAPm $>10 \%$ del valor inicial [i.e., pacientes respondedores, $\triangle \mathrm{PAPm}=-27 \pm 11 \%$ $(-9 \pm 5 \mathrm{mmHg})]$. Cinco pacientes fueron clasificados como no-respondedores $(\triangle \mathrm{PAPm}=-2 \pm 7 \%,-1 \pm 4$ $\mathrm{mmHg}$ ). La estancia en UCI fue de 7 \pm 5 días (1-19), y la hospitalaria 13 días (1-36). No se presentaron complicaciones de sangrado mayor ni de otra índole. La supervivencia a 90 días fue de $100 \%$.

Conclusiones: esta serie observa que la fibrinólisis arterial pulmonar induce una mejoría hemodinámica inmediata en una alta proporción de pacientes con TEPa, con un balance beneficio-riesgo favorable en ausencia de complicaciones atribuibles inmediatas o tardías. (Acta Med Colomb 2019; 44: 17-24).

Palabras clave: embolia pulmonar, fibrinólisis, terapia trombolítica, trombectomía .

\section{Abstract}

Introduction: in patients with acute pulmonary embolism (PE), local arterial fibrinolysis can have a very favorable relationship between benefits and risks.

Objective: to characterize the epidemiological and clinical conditions of patients with intermediate-risk of acute PE who have received urgent fibrinolytic therapy directed by catheter.

Methods: a descriptive study of a series of cases of patients treated at the Cardiovascular Foundation of Colombia (2012-2016 period) with a diagnosis of intermediate-risk PE who received fibrinolytic therapy using a pulmonary catheter.

Results: 27 cases were included ( 26 patients, $57 \pm 20$ years, $52 \%$ women). Confirmation of acute pulmonary embolism was performed by angiotomography. $85 \%$ of the cases showed echocardiographic signs of right ventricular dysfunction, $74 \%$ elevation of troponins, and $82 \%$ elevation of BNP or pro-BNP. The door-needle time from diagnosis to fibrinolysis was $29 \pm 31$ hours (max-min, 2-120). The endovascular treatment included alteplase (total dose, $47 \pm 18 \mathrm{mg}$ ) and mechanical
Dr. Mauricio Orozco-Levi: MSc, PhD. Especialista en Neumología. Jefe Servicio de Neumología, Dra. Melissa Mogollón-Vargas: Médico General. Servicio de Neumología; Dr. Libardo Medina-López: Especialista en Cardiología y Hemodinamia. Servicios de Hemodinamia, Cardiología-Hemodinamia; Dra. Tamara Gorgadze: Especialista en Cardiología. Servicios de Hemodinamia, Cardiología-Hemodinamia; Diana Cáceres-Rivera: Enfermera Jefe. Servicio de Neumología, Centro de Investigaciones, Desarrollo e Innovación; Dr. Rubén Duarte-Reyes: Médico General. Servicio de Medicina Crítica y Cuidados Intensivos; Dra. Alba RamírezSarmiento: PT, PhD. Fisioterapeuta, Doctor en Ciencias de la Salud y de la Vida. Servicio de Neumología Centro de Investigaciones, Desarrollo e Innovación; Dr. Carlos Reyes-Martínez: Especialista en Medicina Interna y Cuidado Intensivo, Servicio de Medicina Crítica y Cuidados Intensivos; Dr. Camilo Pizarro-Gómez: Especialista en Anestesiología, Reanimación y Cuidado Intensivo, Jefe de Servicio de Medicina Crítica y Cuidados Intensivos; Dr. José Federico Saaibi-Solano: Especialista en Medicina Interna y Cardiología-Hemodinamia. Jefe de Servicio de Cardiología y Medicina Interna. Fundación Cardiovascular de Colombia-Hospital Internacional de Colombia. Bucaramanga (Colombia). En nombre de la Red Colombiana de Hipertensión Arterial Pulmonar (HAPred.co).

Correspondencia: Dr. Mauricio Orozco-Levi. Bucaramanga (Colombia).

E-mail: mauricioorozco@fcv.org

Recibido: 01/I/2017 Aceptado: 21/I/2019 
fragmentation-aspiration by pulmonary catheter. $78 \%$ of the patients showed decreases in PAPm> $10 \%$ of the initial value [i.e., responding patients, $\triangle \mathrm{PAPm}=-27 \pm 11 \%(-9 \pm 5 \mathrm{mmHg})]$. Five patients were classified as non-responders $(\triangle \mathrm{PAPm}=-2 \pm 7 \%,-1 \pm 4 \mathrm{mmHg})$. The stay in the ICU was $7 \pm$ 5 days (1-19), and the hospital stay was 13 days (1-36). There were no complications of major or other bleeding. The 90 -day survival was $100 \%$.

Conclusions: this series shows that pulmonary arterial fibrinolysis induces an immediate hemodynamic improvement in a high proportion of patients with intermediate-risk PE with a favorable benefit-risk balance in the absence of immediate or delayed attributable complications. (Acta Med Colomb 2019; 44: 17-24).

Keywords: pulmonary embolism, fibrinolysis, thrombolytic therapy, thrombectomy.

\section{Introducción}

El tromboembolismo pulmonar agudo (TEPa) es la complicación más grave de la enfermedad tromboembólica venosa (ETEV). El TEPa es la tercera causa más frecuente de mortalidad por enfermedad cardiovascular, con 10-17\% de mortalidad atribuible en los siguientes tres meses del episodio agudo. En otros casos la enfermedad puede progresar hacia TEP crónico (TEPc) y generar complicaciones a largo plazo. Entre ellas, la hipertensión pulmonar tromboembólica crónica (HPTEC) implica diversos grados de discapacidad a los pacientes e impone altos costos al sistema de salud.

La presentación clínica del TEPa tiene diversos estadios de gravedad. El riesgo de mortalidad y complicaciones mayores a corto plazo está relacionado directamente con la aparición o empeoramiento de falla ventricular derecha debido a la sobrecarga aguda de presión inducida por la obstrucción vascular pulmonar. Se estima que la presión de la arteria pulmonar se incrementa de forma significativa cuando la masa tromboembólica ocluye aproximadamente entre el 30 y $50 \%$ del área vascular pulmonar transversa, adicionalmente un fenómeno reactivo de vasoconstricción pulmonar generada por la liberación tanto de tromboxano $\mathrm{A}_{2}$ como de serotonina también participa del aumento de resistencia vascular pulmonar. La capacidad del ventrículo derecho para adaptarse ante esta sobrecarga aguda es limitada y si la magnitud de la sobrecarga de presión es muy alta, prolongada o progresiva, se precipita falla de ventrículo derecho debido al disbalance entre aporte y demanda de oxígeno, con progresión a isquemia y lesión miocárdica, y una consecuente disminución de la precarga ventricular izquierda. Esta falla ventricular derecha aguda es la responsable del shock cardiogénico y muerte por TEPa.

$\mathrm{El}$ riesgo de complicaciones mayores (choque, muerte) asociadas al TEPa pueden ser estimadas precozmente mediante parámetros clínicos y escalas predictoras. El mayor riesgo se encuentra en los pacientes con TEPa que presentan hipotensión persistente o shock cardiogénico en el momento del diagnóstico, con una mortalidad inicial estimada de al menos $15 \%$ en la primera hora y que puede alcanzar hasta $50 \%$ en los casos más graves; por fortuna, esta presentación clínica es infrecuente representando menos de $5 \%$ de los casos.
Los pacientes sin hipotensión pueden asignarse a un grupo de bajo riesgo de complicaciones o a un grupo de riesgo intermedio. Actualmente esta asignación en el momento del diagnóstico de TEPa se puede realizar mediante una estrategia compuesta y rápida de (1) imágenes diagnósticas para valorar la presencia de disfunción ventricular derecha (ecocardiografía o tomografía computarizada) (2), biomarcadores séricos de estrés ventricular por sobrecarga mecánica del ventrículo derecho (i.e., elevación de péptidos natriuréticos BNP o NT-proBNP), y (3) biomarcadores de lesión miocárdica (i.e., elevación de la troponina T o I). La presencia de alguno de estos tres criterios identifica a los pacientes de riesgo intermedio, en quienes la mortalidad oscila entre 3 y $15 \%$ a los treinta días del evento.

Los pacientes que no cumplen ningún criterio anterior son clasificados en bajo riesgo, los cuales en general tienen buen pronóstico a corto y largo plazo una vez se han logrado niveles óptimos de anticoagulación con una mortalidad menor a $2 \%$.

Esta estratificación permite identificar al grupo de pacientes que deben recibir recursos terapéuticos más avanzados y adicionales a la anticoagulación. Dos de éstos recursos son la fibrinólisis farmacológica (tanto vía sistémica como intraarterial pulmonar) y la terapia endovascular dirigida por catéter (TDC).

El objetivo de ambas estrategias es disminuir agudamente la resistencia vascular pulmonar, alcanzar una reperfusión pulmonar rápida, facilitar la recuperación funcional del ventrículo derecho, aumentar la perfusión sistémica, mejorar los síntomas, mejorar el pronóstico de supervivencia a corto plazo, disminuir el riesgo de cronicidad de los defectos, y disminuir el riesgo de progresión a HPTEC.

La administración de tratamiento fibrinolítico sistémico está limitada por balance entre los beneficios clínicos y eventos adversos potenciales. Así es el caso de pacientes con TEPa de alto riesgo, en quienes los beneficios de la terapia de reperfusión temprana están ampliamente demostrados pero menos de $30 \%$ de los pacientes la reciben debido a las contraindicaciones, e incluso en ausencia de las mismas, se presentan complicaciones hemorrágicas mayores de hasta $20 \%$ incluyendo un riesgo considerable de hemorragias intracraneales de 3-5\%. Por extensión, el tratamiento 
fibrinolítico de pacientes con TEPa sin-choque ha sido un tema de debate durante varios años. Existen evidencias de que la fibrinólisis como estrategia de reperfusión primaria en TEPa de riesgo intermedio induce beneficios de parámetros hemodinámicos, disminuye el porcentaje de recurrencia, restablece más precozmente el flujo pulmonar, y obtiene mayor recuperación funcional del ventrículo derecho. En el ensayo clínico multicéntrico Europeo PEITHO, el cual contó con la mayor población de casos de TEPa de riesgo intermedio hasta la fecha de nuestro estudio $(n=1006)$, se compararon los efectos y seguridad de la fibrinólisis sistémica adjunta a terapia anticoagulante comparada con la anticoagulación convencional en este tipo de pacientes. Se encontró que el tratamiento fibrinolítico podía disminuir el riesgo de descompensación hemodinámica pero con un impacto desdeñable en el pronóstico de supervivencia. Por el contrario, se observó un aumento en el riesgo de sangrado (tanto fatal como no-fatal) que sobrepasaba la expectativa de beneficios clínicos. Estos resultados permitieron considerar que la fibrinólisis sistémica no debe recomendarse como tratamiento de primera línea de los pacientes con TEPa de riesgo intermedio sino como tratamiento de segunda línea ante un empeoramiento clínico relevante definido en términos de descompensación hemodinámica (i.e., fibrinólisis de rescate).

Por todo lo anterior, existe un amplio interés en incorporar y validar nuevas estrategias terapéuticas de reperfusión pulmonar con mucha mejor relación entre los beneficios y el riesgo de complicaciones. En esta línea, la terapéutica con catéteres pulmonares es especialmente atractiva, pues permite la fragmentación y aspiración de trombos, y eventualmente también instila fármacos fibrinolíticos en menor dosis.

En el presente documento se presenta la caracterización de pacientes con TEPa de riesgo intermedio que han sido llevados a TDC y tratamiento fibrinolítico local pulmonar en nuestro centro, el éxito clínico, la ausencia de mortalidad atribuible al procedimiento y ausencia de hemorragias mayores.

\section{Tipo de estudio}

\section{Métodos}

Descriptivo de serie de casos.

\section{Población y recolección de datos}

Se incluyeron todos los pacientes mayores de 18 años que recibieron atención urgente en un centro de alta complejidad de atención de enfermedades cardiovasculares (Fundación Cardiovascular de Colombia; Floridablanca, Santander) entre 4 de marzo 2012 y 30 de septiembre de 2016 por tromboembolismo pulmonar agudo (TEPa) y que fueron llevados a fibrinólisis intraarterial pulmonar percutánea por el servicio de Hemodinamia. El diagnóstico requirió confirmación mediante angiotomografía pulmonar. La clasificación de TEPa de riesgo intermedio se basó en un baremo multifactorial que incluyó la ausencia de compromiso hemodinámico grave (i.e., shock o hipotensión sistémica sostenida), sintomatología de 15 días o menos, y al menos uno de los tres siguientes criterios: 1. disfunción ventricular derecha evidenciada en ecocardiografía, 2. sobrecarga mecánica de ventrículo derecho definida por elevación de $\mathrm{BNP}$ (punto de corte $>100$ ) o pro-BNP $(>600)$; y/o 3. lesión miocárdica confirmada por elevación de troponina (T o I). Los pacientes se identificaron en la base de datos del Servicio de Neumología de la institución, se revisó la historia clínica de cada uno de ellos para identificar las variables (demográficas, clínicas, tratamiento y desenlaces) con el fin de describir en la población de estudio los hallazgos más frecuentes. Los eventos adversos de interés que pudieran estar relacionados directa o indirectamente con el TEPa o el tratamiento fueron incluidos en el análisis como variables continuas o categóricas, según el caso, y para todo el período de hospitalización o seguimiento (90 días posteriores al ingreso hospitalario). Se definieron como variables de interés: 1. hemorragia menor o mayor (según requiriese intervencionismo específico o transfusión), hemorragia no-grave o grave (según riesgo de invalidez permanente o riesgo vital), 2. necesidad de ventilación mecánica (invasiva o no-invasiva), 3. hemoptisis (amenazante o no-amenazante, según riesgo vital), 4. shock (presión sistólica menor de 90 $\mathrm{mmHg}$ ), 5. necesidad de soporte vasoactivo, 6. trastornos de oxigenación (según relación $\mathrm{Pa} / \mathrm{FiO}_{2}$ menor de 250), 7 . número de días de estancia en UCI, 8. número de días de estancia hospitalaria, 9. recurrencia antes de 90 días, reingreso antes de 90 dias, y 10. muerte.

\section{Consideraciones éticas}

El estudio se desarrolló de acuerdo con lo establecido a la normativa internacional de la Declaración de Helsinki, Reporte de Belmont, Pautas CIOMS (Consejo de Organizaciones Internacionales de las Ciencias Médicas). Atendiendo a la Resolución 008430 (octubre 4 de 1993), la presente se considera una investigación de riesgo mínimo por su carácter descriptivo de serie de casos. El manuscrito fue presentado al Comité de Ética y Técnico Científico de la Fundación Cardiovascular para aprobación de publicación.

\section{Análisis estadístico}

Las variables cuantitativas se expresaron como valores de la media, mediana, desviación típica de la media, valores máximo-mínimo, y/o rangos (máximo-mínimo o intercuartil) según se considerara pertinente. Las variables cualitativas se expresaron en términos de frecuencias absolutas y relativas. Se utilizó el programa SPSS (versión 21) bajo respectiva licencia de uso.

\section{Resultados}

Se identificaron 27 casos (26 pacientes) con TEPa de riesgo intermedio que fueron llevados a fibrinólisis intraarterial pulmonar percutánea en el Servicio de Hemodinamia. 
Aproximadamente la mitad de los pacientes (52\%) fueron remitidos desde otro centro de atención en salud. En $44 \%$ de los casos se cumplieron los tres criterios establecidos para estratificación de la patología como de riesgo intermedio. En $33 \%$ se cumplían dos criterios y en los restantes un criterio. La edad de los pacientes fue de $57 \pm 20$ años, con una relación hombre:mujer de 1:1. En general, el TEPa es causada por la coexistencia de factores de riesgo que se agrupan en circunstanciales, adquiridos o genéticos (trombofilias). El $96 \%$ de los pacientes presentaban uno o más factores predisponentes. Se identificaron como más frecuentes la obesidad, el tabaquismo e historia previa de TVP o TEP. En nuestro estudio, únicamente $4 \%$ de los casos fue considerado idiopático. Si consideramos la exposición a humo de leña como un factor potencialmente relacionado, la proporción de casos idiopáticos sería $0 \%$.

El tiempo previo de síntomas sugestivos de TEPa al momento del diagnóstico fue de 96 \pm 1 horas (rango: 0-20 días). La disnea fue el síntoma predominante en $100 \%$ de los pacientes, seguida de dolor torácico $(78 \%)$, palpitaciones $(26 \%)$ y síncope $(22 \%)$. Las principales comorbilidades fueron hipertensión arterial sistémica $(48 \%$ de los pacientes), enfermedad pulmonar obstructiva crónica (EPOC, en 22\%), HPTEC previa (22\%), y trombosis venosa profunda concomitante $(64 \%)$, muy similar a lo descrito en la literatura. En cuanto a los signos vitales al momento del diagnóstico, se registró una presión arterial sistólica de $128 \pm 17 \mathrm{mmHg}$, frecuencia cardiaca $96 \pm 20$ latidos por minuto, y saturación de oxihemoglobina basal de $90 \pm 4 \%\left(\mathrm{FiO}_{2}=0.21\right)$. En cuanto al intercambio gaseoso, la mayoría de pacientes presentaron alcalosis respiratoria con hipoxemia e hipocapnia, reflejados en valores medios de $\mathrm{pH}$ de $7.41 \pm 01, \mathrm{PaCO}_{2}$ de $33 \pm 8 \mathrm{mmHg}$ e hipoxemia moderada con una $\mathrm{PaFiO}_{2}$ de $233 \pm 96$ (Tabla 1).

El hallazgo radiológico más frecuente fue una radiografía de tórax normal (70\% de los casos). El criterio de inclusión de los pacientes implicaba que la angiotomografía demostrara defectos de llenado en arterias pulmonares. En todos los casos estos defectos afectaban las arterias pulmonares principales. Los defectos fueron más proximales y de mayor proporción en el pulmón derecho. Se evidenció aumento del diámetro ventricular derecho en $85 \%$ de los casos (Figura 1), elevación de marcadores de lesión miocárdica (troponina $\mathrm{T}$ y/o I) en 74\%, y elevación de BNP y/o pro-BNP en $82 \%$.

El 56\% de los pacientes recibió anticoagulación con heparinas de bajo peso molecular (HBPM) inicial. El tiempo desde el diagnóstico de TEP hasta la fibrinólisis fue de 29 \pm 31 horas (rango, 2-120) (Tabla 2). Al inicio del procedimiento hemodinámico, el $85 \%$ de los pacientes mostró hipertensión pulmonar (i.e., PAPm>25 mmHg) (Figura 2). El trombolítico utilizado en todos los casos fue alteplasa, a una dosis promedio $47 \pm 18 \mathrm{mg}$. Al final del procedimiento, la PAPm mostró un cambio de $-27 \pm 11 \%$ del valor inicial (equivalente a $-9 \pm 5 \mathrm{mmHg}$ ). En $72 \%$ de los pacientes, el cambio en la
Tabla 1. Características demográficas y principales antecedentes médicos de los pacientes.

\begin{tabular}{|c|c|}
\hline Casos (n) & 27 \\
\hline \multicolumn{2}{|l|}{ Edad (años) } \\
\hline Media $\pm D E$ & $57 \pm 20$ \\
\hline Mediana (rango intercuartil) & $60(35)$ \\
\hline Mujeres, n (\%) & $14(52)$ \\
\hline $\mathrm{IMC}, \mathrm{kg} / \mathrm{m}^{2}$ & $29 \pm 6$ \\
\hline Historia de Tabaquismo, $\mathrm{n}(\%)$ & $11(40)$ \\
\hline Tabaquismo actual, n (\%) & $5(18)$ \\
\hline Ex tabaquismo, $\mathrm{n}(\%)$ & $6(22)$ \\
\hline Tabaquismo de segunda mano, $\mathrm{n}(\%)$ & $1(4)$ \\
\hline Exposición a humo de leña en domicilio, $\mathrm{n}(\%)$ & $9(35)$ \\
\hline Sin dato $\mathrm{n}^{\circ}(\%)$ & $1(4)$ \\
\hline Índice de Charlson, u.a. & $1,89 \pm 2,0$ \\
\hline Antecedente previo de TVP o TEP, $\mathrm{n}(\%)$ & $5(19)$ \\
\hline Hospitalización en los últimos 3 meses, n (\%) & $0(0)$ \\
\hline Diagnóstico previo de neoplasia, n (\%) & $1(4)$ \\
\hline Neoplasia documentada en el primer año, n (\%) & $1(4)$ \\
\hline Sin dato $\mathrm{n}^{\circ}$ & 6 \\
\hline Diagnóstico previo de trombofilia, $\mathrm{n}(\%)$ & $4(15)$ \\
\hline Trombofilia documentada en el primer año, $\mathrm{n}(\%)$ & $1(4)$ \\
\hline Sin dato $n^{\circ}$ & 8 \\
\hline $\begin{array}{l}\text { Abreviaturas: }(n) \text { : número; }(D E) \text { : desviación están } \\
(T V P) \text { : trombosis venosa profunda; }(T E P) \text { tromboemb } \\
\text { sentan media } \pm D E \text {, salvo que se exprese otra unidad }\end{array}$ & $\begin{array}{l}\text { des arbitrarias; } \\
\text { os valores repre- }\end{array}$ \\
\hline
\end{tabular}

PAPm fue mayor a $10 \%$ de su valor inicial, definido en este caso como grupo de respondedores $(\triangle \mathrm{PAPm}=-27 \pm 11 \%$, equivalente a $-9 \pm 5 \mathrm{mmHg}$ ) (Figura 2). Cinco pacientes fueron considerados no-respondedores atendiendo a que su cambio en la PAPm fue menor de $10 \%$ del valor inicial ( $\triangle \mathrm{PAPm}=-2 \pm 7 \%$, equivalente a $-1 \pm 4 \mathrm{mmHg}$ ) (Figura 3 ). En $26 \%$ de los pacientes se indicó la inserción de filtro de vena cava inferior, sin que se haya retirado hasta la fecha.

Ninguno de los pacientes presentó sangrado mayor asociado al procedimiento. Dos pacientes presentaron un sangrado menor definido como hematoma en el sitio de acceso venoso femoral, uno presentó hemoptisis no-amenazante. La estancia en UCI fue de $7 \pm 5$ días (rango, 1-19). La estancia hospitalaria total fue de $13 \pm 8$ días (rango, 1-36). Al egreso el $48 \%$ de los pacientes continuó anticoagulación con dicumarínicos mientras que los demás recibieron prescripción con nuevos anticoagulantes orales. Un paciente presentó recurrencia embólica en los primeros 90 días. Tres pacientes requirieron reingreso a nuestra institución dentro de los 90 días siguientes por enfermedad general no relacionada (Tabla 3). La mortalidad global a 90 días fue nula $(0 \%)$. 

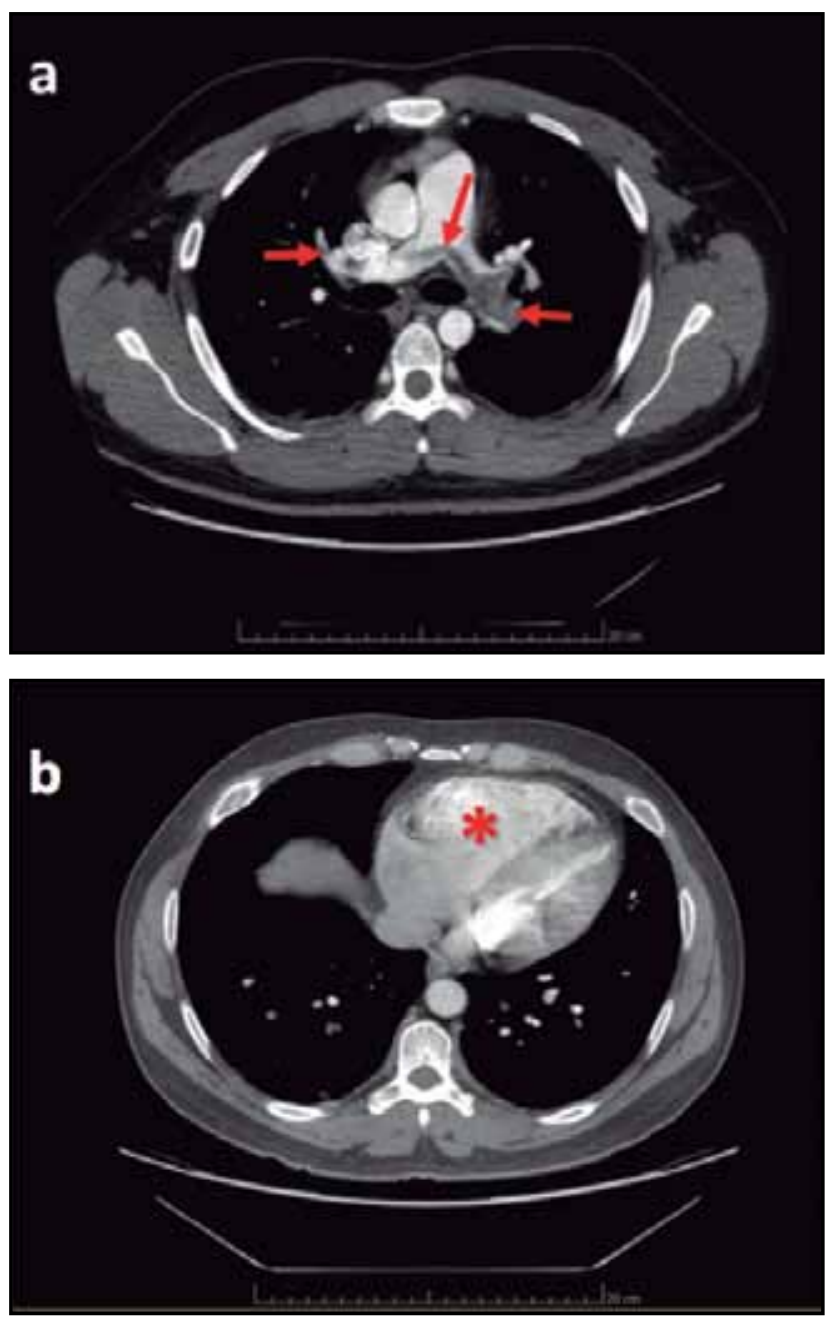

Figura 1. (a): defectos de llenado en bifurcación de arteria pulmonar principal y en arterias lobares, tanto derechas como izquierdas (flechas). (b): aspecto de ventrículo derecho con evidente dilatación, en angiotomografía con contraste.

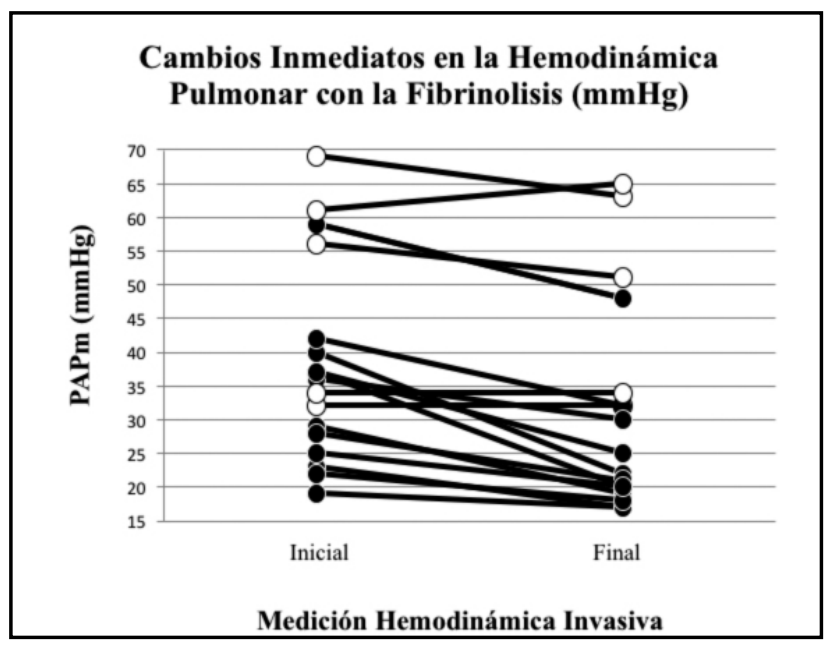

Figura 2. Valores individuales de la presión arterial pulmonar (media) registrados tanto al inicio como al final $(n=18)$ del procedimiento hemodinámico invasivo y administración de fibrinolítico intraarterial pulmonar. Los pacientes respondedores (i.e., cambio $>10 \%$ del valor inicial) aparecen representados con puntos negros (๑), mientras que los norespondedores aparecen con puntos blancos $(\mathrm{O})$. Abreviaturas: (PAPm): presión media de arteria pulmonar.
Tabla 2. Características clínicas de los pacientes al ingreso.

\begin{tabular}{|c|c|}
\hline Casos (n) & 27 \\
\hline Disnea, n (\%) & $27(100)$ \\
\hline Dolor torácico, n (\%) & $21(78)$ \\
\hline Palpitaciones, $\mathrm{n}(\%)$ & $7(26)$ \\
\hline Síncope, n (\%) & $6(22)$ \\
\hline Hemoptisis, n (\%) & $1(4)$ \\
\hline \multicolumn{2}{|l|}{ Horas entre inicio de síntomas y diagnóstico } \\
\hline Media \pm SD & $185 \pm 222$ \\
\hline Mediana (RIQ) & $96(228)$ \\
\hline Presión arterial sistólica, $\mathrm{mmHg}$ & $128 \pm 17$ \\
\hline Frecuencia cardíaca, lpm & $96 \pm 20$ \\
\hline Saturación de oxihemoglobina, $\%$ & $90 \pm 4,0$ \\
\hline $\mathrm{pH}$ & $7,4 \pm 0,1$ \\
\hline $\mathrm{PaO}_{2}, \mathrm{mmHg}$ & $82 \pm 36$ \\
\hline $\mathrm{PaCO}_{2}, \mathrm{mmHg}$ & $33 \pm 8$ \\
\hline $\mathrm{HCO}_{3}, \mathrm{mEq} / \mathrm{L}$ & $19 \pm 3$ \\
\hline Radiografía de tórax normal, n (\%) & $19(70)$ \\
\hline Confirmación de embolia pulmonar por Angio-TC, n (\%) & $27(100)$ \\
\hline Defecto de llenado arterias principales, $\mathrm{n}(\%)$ & $27(100)$ \\
\hline TVP concomitante, $\mathrm{n}(\%)$ & $16(64)$ \\
\hline Sin datos & $2(7)$ \\
\hline $\begin{array}{l}\text { Confirmación de Disfunción ventrículo derecho en ecocar- } \\
\text { diograma inicial, } \mathrm{n}(\%)\end{array}$ & $22(85)$ \\
\hline Sin dato, $\mathrm{n}(\%)$ & $1(4)$ \\
\hline TAPSE, $\mathrm{mm}$ & $17 \pm 6$ \\
\hline PAPs, estimada por ecocardiografía inicial, n (\%) & $66 \pm 28$ \\
\hline Derrame pericárdico, n (\%) & $4(15)$ \\
\hline Troponina T elevada, $\mathrm{n}(\%)$ & $10(37)$ \\
\hline Troponina I elevada, $\mathrm{n}(\%)$ & $11(40)$ \\
\hline Troponina $\mathrm{T}$ o I elevada, $\mathrm{n}(\%)$ & $20(74)$ \\
\hline Elevación de BNP o Pro-BNP, n (\%) & $18(82)$ \\
\hline Sin dato, $\mathrm{n}(\%)$ & $5(19)$ \\
\hline \multicolumn{2}{|l|}{ Tiempo transcurrido entre diagnóstico y fibrinolisis, horas } \\
\hline Media \pm SD & $28 \pm 31$ \\
\hline Mediana (RIQ) & $24(43)$ \\
\hline \multicolumn{2}{|l|}{ Tratamiento con heparina antes de fibrinolisis } \\
\hline No-fraccionada, $(\mathrm{n} \%)$ & $12(46)$ \\
\hline Fraccionada, $\mathrm{n}(\%)$ & $15(58)$ \\
\hline Ninguna, $\mathrm{n}(\%)$ & $1(4)$ \\
\hline \multicolumn{2}{|c|}{$\begin{array}{l}\text { Abreviaturas: }(n) \text { : número; }(D E) \text { : desviación estándar; }(T A P S E) \text { : pico de excursión } \\
\text { sistólica del anillo tricuspídeo; }(B N P) \text { : péptido natriurético cerebral; }(R I Q) \text { : rango } \\
\text { intercuartil. Los valores representan media } \pm D E \text {, salvo que se exprese otra unidad } \\
\text { específicamente. }\end{array}$} \\
\hline
\end{tabular}


Tabla 3. Características hemodinámicas y desenlaces con el tratamiento fibrinolítico mediante catéter pulmonar.

\begin{tabular}{|c|c|}
\hline Casos, $\mathrm{n}(\%)$ & $27(100)$ \\
\hline Dosis total de fibrinolítico (alteplase), mg & $47 \pm 20$ \\
\hline PAPs inicial, $\mathrm{mmHg}$ & $67 \pm 23$ \\
\hline PAPd inicial, $\mathrm{mmHg}$ & $20 \pm 8$ \\
\hline PAPm inicial, mmHg & $37 \pm 14$ \\
\hline PAPs final, $\mathrm{mmHg}$ & $59 \pm 26$ \\
\hline PAPd, final, $\mathrm{mmHg}$ & $16 \pm 10$ \\
\hline PAPm final, $\mathrm{mmHg}$ & $32 \pm 16$ \\
\hline Inserción de filtro de vena cava inferior, n (\%) & $7(26)$ \\
\hline Hipotensión/shock durante el ingreso, n (\%) & $0(0)$ \\
\hline Muerte durante el procedimiento hemodinámico, $\mathrm{n}(\%)$ & $0(0)$ \\
\hline Sangrado mayor, n (\%) & $0(0)$ \\
\hline Sangrado menor, n (\%) & $3(11)$ \\
\hline Días de estancia hospitalaria & $13 \pm 8$ \\
\hline Días de estancia en UCI & $7 \pm 5$ \\
\hline Pacientes con ventilación mecánica, $\mathrm{n}$ (\%) & $3(11)$ \\
\hline Días de ventilación mecánica & $2 \pm 1$ \\
\hline Reingreso a 90 días, n (\%) & $3(11)$ \\
\hline Recurrencia embólica, n (\%) & $1(4)$ \\
\hline Tratamiento anticoagulante al egreso & \\
\hline Warfarina, n (\%) & $13(48)$ \\
\hline Nuevos anticoagulantes, $\mathrm{n}(\%)$ & $11(41)$ \\
\hline HBPM, n (\%) & $3(11)$ \\
\hline Fallecidos a 90 días del evento inicial, n (\%) & $0(0)$ \\
\hline Sin dato $n(\%)$ & $5(18 \%)$ \\
\hline \multicolumn{2}{|c|}{$\begin{array}{l}\text { Abreviaturas: Valores en términos de media } \pm D E \text {, salvo que se exprese otra unidad } \\
\text { específicamente. }(n) \text { : número; }(D E) \text { : desviación estándar; }(P A P s, m, d) \text { : presión arterial } \\
\text { pulmonar sistólica, media y diastólica, respectivamente; }(H B P M) \text { : heparina de bajo } \\
\text { peso molecular. Los valores representan media } \pm D E \text {, salvo que se exprese otra unidad } \\
\text { específicamente. }\end{array}$} \\
\hline
\end{tabular}

\section{Discusión}

El presente estudio describe las características clínicas, funcionales y de seguimiento de los pacientes que recibieron tratamiento endovascular con catéter por tromboembolismo pulmonar agudo (TEPa) de riesgo intermedio en un hospital de alta complejidad en Colombia. Una gran proporción de los pacientes mostraba cifras de presión pulmonar media $>25 \mathrm{mmHg}$ en el momento del cateterismo pulmonar. Una gran proporción mostró cambios hemodinámicos favorables inmediatos. Específicamente se observó que 72\% de los pacientes mostraba mejoría hemodinámica pulmonar precoz. Inclusive, la presión pulmonar se normalizó en seis pacientes. Estos hallazgos positivos son similares a lo repor-
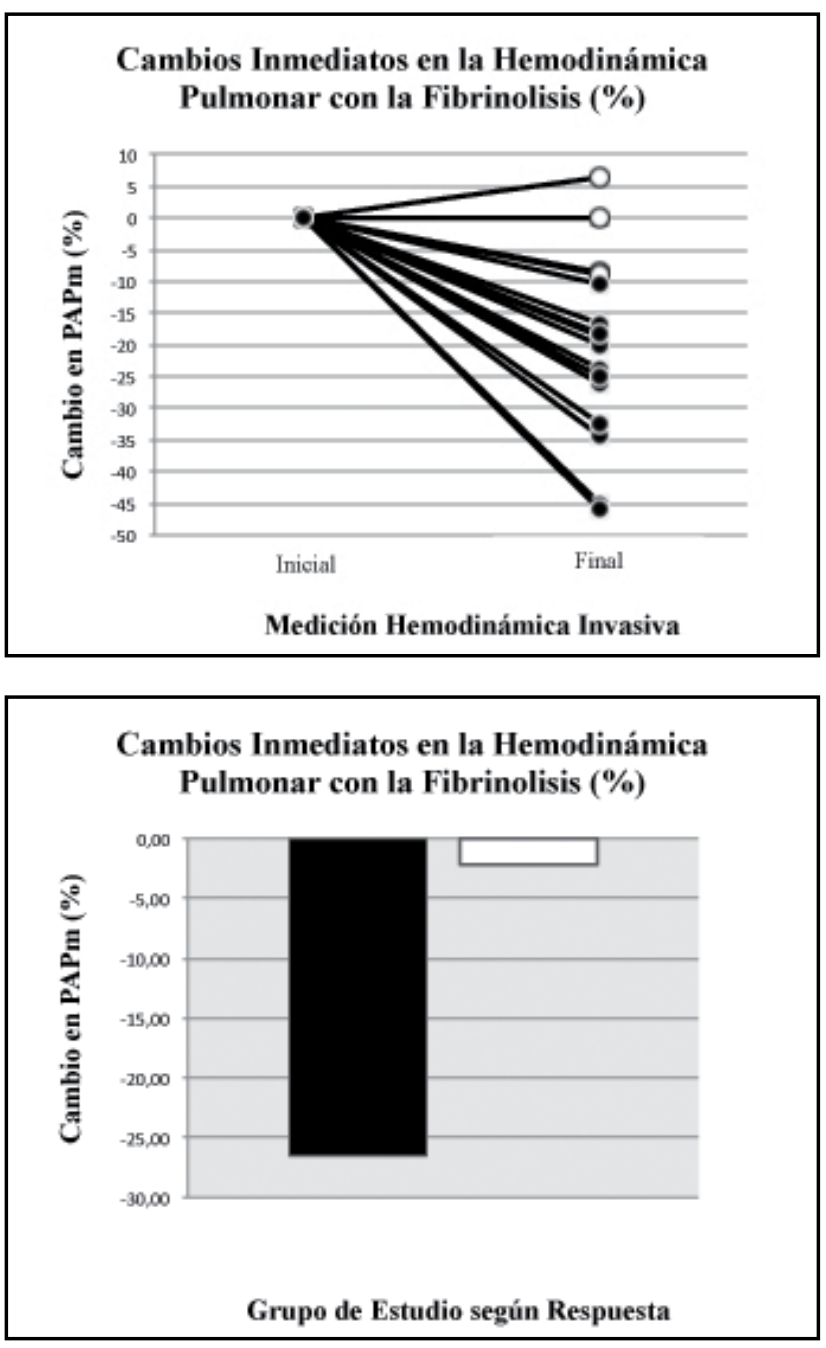

Figura 3. (a) Valores individuales normalizados de la presión media arterial pulmonar en términos de porcentaje respecto del valor inicial registrados tanto al inicio como al final $(n=18)$ del procedimiento hemodinámico invasivo y administración de fibrinolítico intraarterial pulmonar. Los pacientes respondedores (i.e., cambio $>10 \%$ del valor inicial) aparecen representados con puntos negros $(\mathbf{\bullet})$, mientras que los no-respondedores aparecen con puntos blancos (O). (b) Valores medios del cambio (porcentaje) de la presión media de arteria pulmonar en los pacientes respondedores (barra negra) y los no-respondedores (barra blanca). Abreviaturas: (PAPm): presión media de arteria pulmonar.

tado por Latacz et. al. quienes inclusive han evidenciado una disminución de la mortalidad asociada a TEPa como valor añadido de la fibrinólisis intraarterial pulmonar.

Es importante anotar que, sin embargo, cinco pacientes (27\%) no evidenciaron esa mejoría hemodinámica inmediata o precoz. De éstos, dos eran mayores de 75 años, dos cumplían con hipertensión pulmonar tromboembólica crónica de base, y uno sufría de fibrosis pulmonar. No podemos descartar que haya habido mejoría hemodinámica posterior al procedimiento, pues no se mantuvo el catéter pulmonar ni se dispuso de ecocardiografía transtorácica en el periodo mediato. La potencial mejoría a mediano plazo tras la TDC no está bien definida. Coincidimos con Smith et. al. en considerar que la evolución a largo plazo ha sido poco estudiada, pero en su experiencia, el seguimiento a seis meses puede 
mostrar que los desenlaces son mejores al comparar con los pacientes que no reciben esta estrategia terapéutica.

Los eventos adversos asociados al procedimiento fueron prácticamente nulos, excepto por dos hematomas en el sitio de punción y hemoptisis menor autolimitada en un caso. La supervivencia a 90 días ha sido de $100 \%$ en todos los casos con posibilidad de seguimiento (cinco casos no pudieron ser contactados por cambio de número telefónico o domicilio).

La fibrinolisis como tratamiento del TEPa de alto o mediano riesgo tiene un amplio interés clínico. Desde el punto de vista conceptual, una estrategia de reperfusión ideal para el tratamiento del TEPa debe ser eficaz para revertir la disfunción ventricular derecha y sus consecuencias, pero suficientemente segura de tal forma que el riesgo de complicaciones sea equivalente al tratamiento anticoagulante convencional, como lo sugiere el American College of Chest Physicians.

En este sentido, la fibrinólisis sistémica primaria tiene una relación riesgo-beneficio favorable en TEPa de alto riesgo, pero desfavorable en el de riesgo intermedio. Esta limitación no está relacionada con la eficacia trombolítica en sí misma que de hecho es extremadamente alta, sino a la seguridad de la dosis sistémica total en términos de incremento del riesgo hemorrágico grave (intracraneal o extracraneal).

En este escenario, la TDC con fibrinolisis local puede tener un papel preferente y de hecho se utiliza cada vez con mayor frecuencia en varios centros hospitalarios, inclusive en situaciones clínicas en las cuales no se había considerado previamente como la presencia de grandes masas trombóticas (i.e., oclusión de 50\% de vascular pulmonar) o hipoxemia grave persistente.

Un metaanálisis en pacientes con TEPa de alto riesgo ha establecido que la TDC tiene un éxito clínico (definido como estabilización hemodinámica, resolución de la hipoxemia, y aumento de supervivencia de los pacientes durante la hospitalizac) del $86 \%$ frente a complicaciones hemorrágicas (menores y mayores) de 7 y $2 \%$, respectivamente.

Estos resultados fueron altamente favorables al comparar con la eficacia (77\%) y riesgo de hemorragia mayor (22\%) de la fibrinólisis sistémica reportadas en el International Cooperative Pulmonary Embolism Registry (ICOPER) ${ }^{6}$. Por estos argumentos y ante estas evidencias, la TDC es recomendada actualmente como la primera línea de tratamiento en pacientes con TEPa de alto riesgo con contraindicación para un tratamiento fibrinolítico sistémico. Sin embargo, en pacientes con TEPa de riesgo intermedio la evidencia no es tan clara para hacer una recomendación a favor de la TDC, lo cual es la principal limitante para ampliar su uso. La mayor parte de la información referente a los efectos de la TDC está representada por series de casos como la que presentamos en este artículo y está restringido a centros con posibilidad de técnicas diagnósticas y terapéuticas hemodinámicas invasivas. No existen ensayos clínicos aleatorizados con tamaños muestrales amplios que comparen la TDC con la trombólisis sistémica, por lo cual esta técnica no cuenta aún con aproba- ciones por agencias reguladoras nacionales o internacionales. Otro punto por resaltar es la ausencia de guías normalizadas en las cuales estén definidas con claridad las especificaciones de experticia o especialización del personal idóneo para su realización, características técnicas del procedimiento o consideraciones metodológicas y administrativas para la subvención del mismo por el sistema de salud público.

Teniendo en cuenta tanto los datos de nuestra serie junto con la literatura publicada, debemos hacer algunos comentarios respecto de las ventajas intrínsecas a la TDC en pacientes con TEPa de riesgo intermedio. La característica más atractiva de los regímenes de tratamiento endovascular (mecánico, farmacológico o combinado) es que ofrecen la posibilidad de obviar o disminuir ampliamente las dosis efectivas del fármaco fibrinolítico, reduciendo así el riesgo hemorrágico. En la literatura se han descrito diferentes técnicas de TDC, algunas de las cuales incluyen trombectomia por aspiración (catéter de succión Greenfield®), trombectomía por fragmentación (catéter-balón de angioplastia pig tail ${ }^{\circledR}$ ), y trombectomia hidrodinámica (catéter Hydrolyzer® o Angiojet $\left({ }^{\circledR}\right)$ principalmente. Algunos de ensayos clínicos que han evaluado el tratamiento convencional (anticoagulación) como comparador ante la terapia endovascular pulmonar (i.e., reolisis por ultrasonido) en pacientes con TEPa de riesgo intermedio, han evidenciado que el tratamiento endovascular induce mejoría en las variables ecocardiográficas sin complicaciones hemorrágicas mayores.

Nuestra técnica ha estado basada tanto en el uso de un catéter multipropósito y pig-tail para la fragmentación y aspiración con vacío generado manualmente, o catéteres de presión controlada automáticamente (dispositivo Penumbra $\left.{ }^{\circledR}\right)$.

Lo anterior tiene varias ventajas tales como, primero, representar componente tanto diagnóstico como terapéutico y los efectos del tratamiento pueden ser monitorizados en tiempo real por medio de la angiografía; segundo, la embolectomía por fragmentación y aspiración mecánica pueden evitar o resolver rápidamente el shock al lograr la fragmentación inmediata del trombo oclusivo en la circulación pulmonar principal; tercero, después de la fragmentación, una mayor superficie del trombo puede estar expuesta a los agentes trombolíticos requiriendo una dosis de infusión más baja, ya que el agente fibrinolítico estará concentrado en la masa trombótica y mucha menor proporción en la circulación sistémica, requiriendo adicionalmente menos tiempo necesario para lograr la trombolisis.

Finalmente, las principales limitaciones que merecen ser mencionadas en relación con el presente estudio tienen que ver con su carácter descriptivo, ausencia de tratamiento comparador, ausencia de aleatorización en la asignación al tratamiento, y su carácter unicéntrico.

\section{Conclusión}

El tratamiento endovascular pulmonar con fibrinolisis a dosis menores (medias o inferiores) aparenta ser un trata- 
miento útil en casos de tromboembolismo pulmonar agudo de riesgo intermedio. Las complicaciones mayores han sido nulas y la supervivencia a 90 días de seguimiento ha sido de $100 \%$. Desde nuestra experiencia consideramos que está justificado realizar ensayos clínicos multicéntricos diseñados específicamente para evaluar la eficacia y seguridad de la TDC en pacientes que sufren la devastadora TEPa.

\section{Referencias}

1. McCumber M, Hylek EM, Buller H, Weitz JI, Raskob Gl. http://www. ncbi.1. McCumber M, Hylek EM, Buller H, Weitz JI, Raskob GI. ISTH Steering Committee for World Thrombosis Day. Global public awareness of venous thromboembolism. J Thromb Haemost. 2015 Aug;13(8):1365-71.

2. Yang $\mathbf{S}$, Yang $\mathbf{Y}$, Zhai Z, Kuang T, Gong J, Zhang S. et al. Incidence and risk factors of chronic thromboembolic pulmonary hypertension in patients after acute pulmonary embolism. Journal of Thoracic Disease. 2015;7(11):1927-1938.

3. Wood KE. Major pulmonary embolism: review of a pathophysiologic approach to the golden hour of hemodynamically significant pulmonary embolism. Chest. 2002;121:877-905

4. Kucher N, Rossi E, De Rosa M, Goldhaber SZ. Massive Pulmonary Embolism. Circulation. 2006;113:577-582.

5. Goldhaber SZ, Visani L, De Rosa M. Acute pulmonary embolism: clinical outcomes in the international cooperative pulmonary embolism registry (ICOPER). Lancet 1999; 353: 1386-1389.

6. Sanchez O, Trinquart L, Colombet I, Durieux P, Huisman MV, Chatellier G, et al. Prognostic value of right ventricular dysfunction in patients with haemodynamically stable pulmonary embolism: A systematic review. Eur Heart J. 2008; 29:1569-77.

7. Jiménez D, Aujesky D, Yusen RD. Risk stratification of normotensive patients with acute pulmonary embolism. Br J Haematol. 2010; 151: 415-24.

8. Lankeit M, Gomez V, Wagner C, Aujesky D, Recio M, Briongos S, et al. A strategy combining imaging and laboratory biomarkers in comparison to a simplified clinical score for risk stratification of patients with acute pulmonary embolism. Chest 2011;141:916-92.

9. Kearon C, Akl EA, Ornelas J, et al. Antithrombotic therapy for vte disease: chest guideline and expert panel report . Chest 2016;149(2):315-352.

10. Konstantinides SV1, Wärntges S. Acute phase treatment of venous thromboembolism: advanced therapy. Systemic fibrinolysis and pharmacomechanical therapy. Thromb Haemost. 2015 Jun;113(6): 1202-9.

11. Marti C, John G, Konstantinides S, Combescure C, Sanchez O, Lankeit M. et al. Systemic thrombolytic therapy for acute pulmonary embolism: a systematic review and meta-analysis. Eur Heart J 2015;36(10):605-614.
12. Mostafa, Ashraf, MD. Alexandros Briasoulis, MD, PhD- Tesfaye Telila, MD. Kevin Belgrave, M. Cindy Grines. MD Treatment of Massive or Submassive Acute Pulmonary Embolism With Catheter-Directed Thrombolysis. Am J Cardiol 2016; 117(69): 1014-1020.

13. Stein PD, Matta F. Thrombolytic therapy in unstable patients with acute pulmonary embolism: saves lives but underused. Am J Med 2012; 125(5): 465-470.

14. Meyer G, Vicaut E, Danays T, Agnelli G, Becattini C, Beyer-Westendorf J, et al. Fibrinolysis for Patients with Intermediate- Risk Pulmonary Embolism. $N$ Engl J Med. 2014;370: 1402-11.

15. Chatterjee S, Chakraborty A, Weinberg I, et al. Thrombolysis for pulmonary embolism and risk of all-cause mortality, major bleeding, and intracranial hemorrhage: a meta-analysis. JAMA 2014; 311:2414.

16.Piazza G, Hohlfelder B, Jaff MR, et al. A Prospective, Single-Arm, Multicenter Trial of Ultrasound-Facilitated, Catheter-Directed, Low-Dose Fibrinolysis for Acute Massive and Submassive Pulmonary Embolism: The SEATTLE II Study. J Am Coll Cardiol Intv. 2015;8(10):1382-1392.

17. Kuo WT, Banerjee A, Kim PS, et al. Pulmonary embolism response to fragmentation, embolectomy, and catheter thrombolysis (PERFECT): initial results from a prospec- tive multicenter registry. Chest. 2015;148:667-73

18. Latacz,Pawel, et al. "Endovascular thrombectomy with the AngioJet System for the treatment of intermediate-risk acute pulmonary embolism: a case report of two patients". Postepy $w$ Kardiologii Interwencyjnej= Advances in Interventional Cardiology 12.1 (2016): 61.

19. Smith, J. Gustav, et al. "Acute right ventricular failure caused by concomitant coronary and pulmonary embolism: successful treatment with endovascular coronary and pulmonary thrombectomy." European Heart Journal: Acute Cardiovascular Care 2.2 (2013): 131-136.

20.Pelliccia, Francesco, et al. "Treatment of acute pulmonary embolism: update on newer pharmacologic and interventional strategies." BioMed research international 2014 (2014).

21. Nakamura S1, Takano H, Kubota Y, Asai K, Shimizu W. Impact of the efficacy of thrombolytic therapy on the mortality of patients with acute submassive pulmonary embolism: a meta-analysis. J Thromb Haemost. 2014 Jul;12(7):1086-95

22. Akin H,Al-Jubouri M,Assi Z, et al. Catheter-directed thrombolytic intervention is effective for patients with massive and submassive pulmonary embolism. Ann Vasc Surg 2014; 28:1589.

23. Kuo WT, Gould MK, Louie JD, Rosenberg JK, Sze DY, Hofmann LV. Catheterdirected therapy for the treatment of massive pulmonary embolism: systematic review and metaanalysis of modern techniques. $J$ Vasc Interv Radiol. 2009; 20: 1431-1440

24. Engelberger RP, Kucher N. Ultrasound-assisted thrombolysis for acute pulmonary embolism: a systematic review. Eur Heart J 2014; 35: 758-764

25. Mogollòn M, Medina L, Gordgadze T, Saaibi JF, Orozco-Levi M. Tratamiento urgente de la embolia pulmonar aguda mediante el sistema de aspiración por catéter Penumbra. Acta Colomb Cuid Intensivo. 2016; 16(1): 59-65. 